\title{
Erratum: Computerized Cardiotocography Analysis: Comparison among Several Parental Ethnic Origins
}

\section{Análise de cardiotocografia computadorizada: comparação entre diversas origens étnicas}

Mariarosaria Di Tommaso ${ }^{1}$ Giulia Martello ${ }^{1}$ Tomi Kanninen $^{1} \quad$ Federica Perelli $^{1}$ Laura lannuzzi ${ }^{1}$ Giovanni Sisti ${ }^{1}$

Rev Bras Ginecol Obstet 2017;39:90.

Address for correspondence Mariarosaria Di Tommaso, MD, PhD, Department of Health Sciences, Università degli Studi di Firenze, Viale Morgagni 84, 50134, Firenze, Italy

(e-mail: mariarosaria.ditommaso@unifi.it).

ERRATUM

Rio de Janeiro, March 3, 2017

Dear readers,

In the article Computerized Cardiotocography Analysis: Comparison among Several Parental Ethnic Origins, published in Rev Bras Ginecol Obstet. 2016;38(12):589-592 (doi: 10.1055/ s-0036-1594288), where it reads:

Di Tommaso Mariarosaria, ${ }^{1}$ Martello Giulia, ${ }^{1}$ Kanninen Tomi, ${ }^{1}$ Perelli Federica, ${ }^{1}$ Iannuzzi Laura, ${ }^{1}$ Sisti Giovanni ${ }^{1}$

It should read:

Mariarosaria Di Tommaso, ${ }^{1}$ Giulia Martello, ${ }^{1}$ Tomi Kanninen, ${ }^{1}$ Federica Perelli, ${ }^{1}$ Laura Iannuzzi, ${ }^{1}$ Giovanni Sisti ${ }^{1}$ 